\section{MONOGRAPHIAE BIOLOGICAE}

VOLUME 72

Series Editors

H.J. Dumont and M.J.A. Werger

\section{Biogeography and Ecology of}

\section{Turkmenistan}

\author{
Edited by \\ VICTOR FET \\ Dept. of Biological Sciences, Loyola University, New Orleans, Louisiana, USA \\ and \\ KHABIBULLA I. ATAMURADOV \\ Natural Conservation Society, Ashgabat, Turkmenistan
}

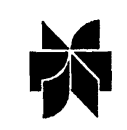

Kluwer Academic Publishers

Dordrecht / Boston / London 
paulus. which are found in plains from the Caspian Sea to Mongolia; another group includes species that inhabit only Middle Asian deserts: Acomthac/isis curvispura. Nohovens crucifer, Aspoeckiana candara, A. longiventris, Lopezus antummalis, L. karakumicus, Maracanda talitzkii, and Quimemurus me'umerus. Two species, Aspoeckiana carlic and Lopezus namus, are endemic for the East Karakum Desert.

A representative East Karakum ant-lion complex from the Repetek Reserve

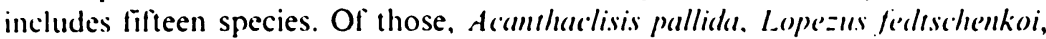

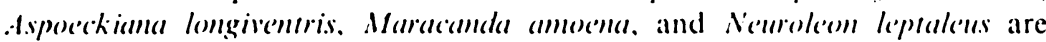
common species attracted to the light at night from May to August. From August to October, Aspoeckiana c'ancluta. Lope'zus antmumalis, Ge'pria lepiclula, and Quinemurus metamerus can be found. Mrrmeccaehurus varians and Nohoverus crucifer have evening activity and fly above the sand at low altitude. In the sand, one can find numerous pits of ant-lion larvae of Morter hralimus. Other pits made by Morter semigriseus larvae are located inside rodent holes but also can be found in human settlements, in cellars and under sheds. Larvac of

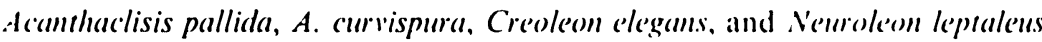
are found in rodent burrows.

\section{Molumtuins and Foothills}

The distinctive ant-lion fauna of the mountains of Turkmenistam falls into two groups: Irano-Turanian species (Palpares solidus, Myrmecuelurus pug/mmanus,

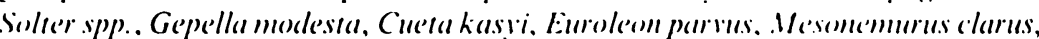
11. sp. De.lfime'us intricatus. and species of Nenrole(om) and Ancient

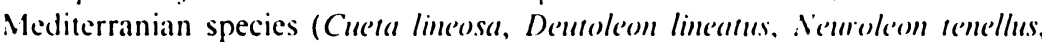
.'icarimus poecilopterus, Nedroleon maculatus, and Megistopus flavicormis).

Numerous flying Mrrmecaelurus paghmamus and Ciretu lineosu have been recorded during the daytime in the Kopetdagh Reserve. Flying species of Mesomemurus and Creoleon spp. appear during the afternoon. Pits of the fiuroleon parvus larvae are found in the sand under the rocks.

River l'alle's:s and Seashore

Because sands encroach upon river banks, many ant-lion species thatt are specific for the deserts (such as species of l.ope zus and Quime'mumus me'tumerus) are found in river valleys. Certain mountain species (e.g.. Cincela lineosa and species of Neuroleon spp. ete.) can disperse from the mountains along the river valleys.

Complexes of ant-lions in river valleys of Turkmenistan are different from those in both desert and mountains. In the sandy banks of the Amudarya River


under the bushes of Tamarix spp. are found pits of larvae of Cincta lineosa and Morter hyalinus. In the evening fly Myrmecaclurus varians and Creoleon spp. fly over the sand dunes in the Tedzhen River valley near Serakhs, whereas Mrrmecaelurus paghmanus and Cueta lineosa are active at dawn. Finally, only on the Caspian Sea shore and islands are found numerous Nohoverus zigan, which are widespread in the North Palearctic.

\section{Fauna and Zoogeography of Spiders (Aranei) of Turkmenistan}

\section{KIRILL G. MIKHAILOV AND VICTOR FET}

\section{Abstract}

In Turkmenistan, 335 species of spiders (Aranei) belonging to 162 genera and 38 families are found. Data on their distribution are given for Turkmenistan and worldwide. The most diverse spider families are Salticidae (62 species), Gnaphosidae ( 52 spp.), Linyphiidae (33 spp.), Thomisidae (28 spp.), Lycosidae (26 spp.), and Araneidae (24 spp.). Two new combinations are proposed: Orthobula charitonovi (Mikhailov, 1986), comb. nov. (= Trachelas charitonovi Mikhailov, 1986) (Liocranidae), and Psammitis turanicus (Charitonov, 1969), comh. nor. (= Xisticus turanicus Charitonov, 1969) (Thomisidae). Distribution of spider species within the republic is influenced primarily by the diverse landscape structure. A specific and impoverished xerophilic fauna of lowland deserts, including the great sand desert of Karakum, is almost emtirely different from the rich mesophile spider fauna found in the Kopetdagh Mountains. Zoogeographic analysis reveals details of origin and possible directions of dispersal of spider fauna. The unique historical role of the Kopetdagh region is discussed; this area could have served as an important island/peninsular corridor before the Tethys Sea recession (Oligocene). With mountain uplift and aridization in the Pliocene, Kopetdagh became a sublatitudinal dispersal pathway for mesophilic spiders. About half of araneofauna is represented by widely distributed species; of the other half, such zoogeographic groups as Iranian, Iranian-Turkestanian, European, European-Caucasian, and European-Mediterranean comprise the majority of the mountain araneofauna. Turanian desert species are predominant in the lowland deserts. A number of spider species are currently known only from Turkmenistan, but the degree of local endemism cannot be estimated since the araneofaunas of the adjacent Middle Asian republics, Iran, and Afghanistan are poorly known. 
Introduction

In his 1878 treatment of arachnids from the Calleasus. Ludwig Koch described several forms from the other side of the Caspian Sea - namely from Kratsnovodsk, then a new Russian colonial settlement in the deserts of Transcaspia (Koch 1878). The first detailed study of spiders from Turkmenistan was begun by the famous French arachnologist Eugene Simon. who described a number of new species collected in 1886 by G. Radde. $A$. Walter, and $A$. Konchin during one of the first zoological expeditions to the former Tratrsciaspian Region (Simon 1889. 1899). Part of this material is deposited in Simon 's collection in Museum National d'Histoire Naturelle. Paris. France: several specimens are also deposited in the State Museum of (icorgia fformer (alucasian Museum), Tbilisi, Republic of Georgia.

Turkmenian spider species known by the 1930s were included in the only caltaloguc of USSR spiders compiled by D.E. Kharitonos (19.32. 19.36). In the 19.3()s extensive spider collections were organized by $Y a$ a. Vlasov and identilied by V. Sytshevskaya (Pereleshina) (Vlasov 1937a,b.c; Vlasov and Sytscherskatyal 1937). Materials of V. Sytschevskaya s expedition (o) Turkmenistan in 1924). deposited in the Zoological Museun of Moscow State University (Moscow. Russia). remained unidentified until the $1980 \mathrm{~s}$

A number of new species from Turkmenistan were described by S. Spassky (1934. 1936, 1937, 1939, 1941). Some unpublished datal based on Spassky personal collection (now in Zoological Institute, St. Petershurg) are included in this paper. Spassky (1952) included all existing data on spiders of Turkmenistan in his zoogeographic analysis of the Middle Asian ("Turanian Zoogeographic Province") araneofauna. Fragmentary faunistic data from Turkmenistan were published by the arachnologists of Perm University (Kharitonov 1955: (lachkin 1956, 1960a,b,c, 1964, 1968) and, later, by Bakhvaloy (1978) and Sternbergs (1979). More regular collections in this republic started in the 1970) under the direction of the Zoological Institute (St. Petersburg. then I.eningrad Russia). Faunistic data and descriptions of new species were published specilically on Turkmenistan (Ovtsharenko and Fet 1980): Fet 1982. 1983, 1984b.c.c, 1985a, 1986, 1993; Kuznetsov and Fet 1982. 1986; Dunin and Fet 1985; Nenilin and Fet 1985; Zonshtein and Fet 1985; Mikhailov and Fet 1986: Tanasevitch and Fet 1986) or were incorporated into broader faunistic publications (Dunin 1985, 1990, 1992; Nenilin 1984a,b, 1985; Zonshtein 1985. 1987: Zyuzin 1985; Nenilin and Pestova 1986; Mikhailov 1986. 1987, 199?: Tanasevitch 1989; Platnick and Ovtsharenko 1991: Marusik and Logunov 1990): (Ovisharenko. Platnick, and Song 1992). A number of ecological works also hate been recently produced specifically focused on the spider species of this area (kaplin 1975; Fet 1984d, 1985b: Atamuradov and Sukh 1985: Krivokhatsky and Fet 1981, 1982; Kuznetsov 1985a,b). Other works mention certain spider species and give notes on their ecology (Sabirova 1975. 1977. 1981. 1985, 1986, 1989; Kaplin 1978; Soyunov 1979; Krivokhatsky 1981. 1982a.b, 1983, 1985a,b).
This work is designed to serve as a catalog of spiders from Turkmenistan as well as a review of their distribution with some zoogeographic observations. We incorporated all known literature, as well as some unpublished data from collections of the Zoological Museum of Moscow State University (Moscow, Russia) and the Zoological Institute of the Russian Academy of Sciences (St. Petersburg, Russia) (all spider collections from Turkmenistan compiled by V. Fet from 1975 to 1987 are deposited in these two institutions). During the many years of faunistic and taxonomic studies, numerous type specimens have been analyzed and revised (including those of E. Simon, O. Pickard-Cambridge, S. Spassky, D. Kharitonov, and V. Sytshevskaya). Identification was conducted using modern taxonomic revisions and consultations with available experts and included analysis of external and internal genitalia of adult males and females. Of significant help have been works of Denis (1958) and Roewer (1955, 1960, 1961a,h) on Alghanistan and Iran, and Andreeva (1976) on Tajikistan, all of which include many spiders found also in Turkmenistan. In placement, synonymy, and taxonomy of spiders we followed recent catalogs by Brignoli (1983) and Platnick (1987) as well as classic works of Bonnet (1937-1961) and Roewer (1942, 1954, 1955). Bibliographic references for this paper were compiled using the database "Spider Literature: A Computerized Bibliography Version 1.0," edited by J.A. Coddington (Smithsonian Institution, Washington, DC. USA). Both authors contributed to this project by submitting all known Russian references

The list below includes names and data on distribution (within Turkmenistan and worldwide) of 343 species of spiders belonging to 164 genera and 38 familics. This list undoubtedly will be extended in the future. The most diverse spider families in the Turkmenistan fauna are Salticidae (62 species), (inaphosidae (54 spp.), Linyphiidae (33 spp.), Thomisidae (28 spp.), Lycosidae (26 spp.). and Araneidae (24 spp.).

\section{A List of Spiders of Turkmenistan}

Farm. Alypidas

Alypus murulis Bertkau, 1890. Kopetdagh (Southwest and Central). Mediterranean species.

Fam. Diphuridue

Phyrioschema raddei Simon, 1889. Krasnovodsk, Kopetdagh (Southwest and (entral), Serakhs, Badghyz, Repetek. Iranian species. 
Fam. Nemesiidae

Nemesia hirulai (Spassky, 1937). Kopetdagh (Southwest. Central, and Fast). Serakhs, Badghyz. Iranian species.

Rareniola fedotovi (Charitonov, 1946). Kopetdagh. Iranian species

R. Kopredughensis (Fet, 1984). Kopetdagh (Southwest and Central). Iranian species.

R. redikorzeri (Spassky, 1937). Badghyz, Serakhs. Iranian species.

\section{Fim!. Filistatidae}

Filistata insidiatrix (Forskäl, 1775). Kopetdagh (Southwest, Bakharden Cave) Mediterranean and Afrotropical species.

F. sp. Badkhyz. Iranian species (?).

Pritha crosbyii (Spassky, 1938). Kopetdagh (Southwest). Irano-Turkestanian species.

Finm. Sivrodiclae

Siltodes bertheloti Lucas, 1839. Madau. Serakhs District. Repetck. Mediterranean species.

S. stromdi Spassky, 1941. Kopetdagh (Southwest), Ashkhabad, Badghyz. IranoTurkestanian species.

S. univitutus Simon, 1882. Aidere. Paleotropical species.

Firin. Lo.roscelidae

Lovosceles rufescens (Dufour, 1820). Madau, Bugdaily, Kopetdagh (Southwest and Central), Bakharden, Ashkhabad. Cosmopolitan species.

Firm. Pholcicluse

Hrema transceaspica Spassky, 1934. Krasnovodsk. Kopetdagh (Southwest and (entral). Ashkhabad, Serakhs, Badghyz. Repetek. Farah. Turanian desert specicis (?).

(iratopholeus maculipes Spassky, 1934. Krasnovodsk. Ashkhabad. Badghyz. Repetek. Iranian species.

Hoplopholcus forskali (Thorell, 1871). Ashkhabad. MediterraneanTurkestanian species.

Pholcus nenjukovi Spassky, 1936. Kopetdagh (Southwest). Iranian species.
Fam. Dysderidae

Disiclera aculeara Croneberg, 1875. Krasnovodsk, Kurum, Tekke, Kopetdagh (Southwest and Central), Ashkhabad, Badghyz, Repetek. Turanian desert species ('?).

D. limitcme'a Dunin, 1985. Known only from Badkhyz. Turanian desert species.

D. pococki Dunin, 1985. Known only from Kizyl-Atrek (may also be present in northeastern Iran). Turanian desert species.

D. Kugitungica Dunin, 1992. Known only from Kugitang. Turkestanian species.

D. Iransccispica Dunin et Fet, 1985. Known only from southern Turkmenistan (Southwest Kopetdagh, Ashkhabad, Badghyz). Iranian species.

Fam. Oonopicke

Disclerina loricata (Simon, 1873). Ashkhabad, Repetek. EuropeanMediterranean species.

Famm. Palpimanidae

Palpimumus sogclianus. Charitonov, 1946. Kopetdagh (Southwest), Ashkhabad, Badghyz, Repetek. Iranian species.

Farm. Mimeticke

Ero furcata (Villers, 1789). Kopetdagh (Southwest). Holarctic species.

Mimetus laevigatus (Keyserling, 1863). Kopetdagh (Southwest), Repetek. Ancient Mediterranean species.

Famm. Eresidae

Eresus cinnuherimus (Olivier, 1789).

E. niger Petagna, 1787, nomen praeoccupatum (Merett \& Millidge 1992). E. niger rotundic'ps Simon, 1873.

Kopetdagh (Southwest, Central, and East), Badghyz. Trans-Palearctic species (eastward to South Korea).

Stegodyphus linceutus (Latreille, 1817). Krasnovodsk, Kopetdagh, Akhal-Teke, Ashkhabad, Badghyz. Mediterranean species (eastward to Tajikistan).

Fimm. Oecobiidae (= Urocteidae)

Oecobius nadice (Spassky, 1936). Krasnovodsk, Kopetdagh (Southwest, Central, and East), Ashkhabad, Badghyz, Repetek. Irano-Turkestanian species.

O. tadzhicus Andreeva et Tystshenko, 1969. Kopetdagh (Southwest, Central, and East). Irano-Turkestanian species 
Uroctea limbata (C.L. Koch, 184.3). Kopetdagh (Southwest and Central), Badghyz. Ancient Mediterranean species.

Fam. Hersiliidae

Hersiliola afghanica Roewer, 1962. Kopetdagh (Southwest and Central), Badghyz. Iranian species.

H. sp. n. I. Kopetdagh (Southwest; foothills). A new species which has not been yet described. Iranian species.

II. sp. n. 2 Tuarkyr, Ashkhabad, Repetek, Badghyz. A new species previously listed as H. maculata (Dufour, 1831) (Fet 1983, Krivokhatsky and Fet 1982). Turanian desert species.

Fam. Uloboridae

Uloborus plumipes Lucas, 1846. Kopetdagh (Southwest). MediteraneanHimalayan species.

Ulohorus walckenaerius (Latreille, 1806). Kopetdagh (Southwest and Central), Ashkhabad, Mary (= New Merv), Badghyz. Trans-Palearctic species.

Fam. Theridiidae

Finoplognatha testacea Simon, 1884. Kopetdagh (Southwest and Central). Mediterranean species.

E. thoracica (Hahn, 1831). Kopetdagh (Southwest). Furopean-Mediterranean species.

Faryopis laeta (Westring. 1861). Kopetdagh (Southwest and Central), Badghyz. European-Ancient Mediterranean species

I.: quinumeguttatu Thorell. 1875. Kopetdagh (Southwest). EuropeanMediterranean species.

Latrodectus tredecimgutatus (Rossi, 1790). Meshed-Messerian Plain, Kopetdagh (Southwest and Central), Ashkhabad, Gyuaurs District, Badghyz, Repetek, Kunya-Urgench, Tashauz Oasis. Ancient Mediterranean species.

L. pallidus O. Pickard-Cambridge, 1872. Chilmanedkum Sinds, Kopeldagh (Southwest and Central), Kizyl-Arvat District, Geok-Tepe District, Ashkhabad, Gyuaurs District. East Mediterranean species.

Steatoda albomaculata (De Geer, 1778). Krasnovodsk. Kopetdagh (Central). Ashkhabad, Badghyz, Repetek. Holaretic species.

S. bipunctata (L.. 1758). Kopetdagh (Central). Holaretic species.

S. castanea (Clerck, 1757). Kopetdagh (East). European-Siberian species.

S. grossa (C.L. Koch, 1838). Gasan-Kuli, Sharlouk, Kopetdagh (Southwest and

Central), Ashkhabad, Badghyz, Repetek. Cosmopolitan species.

S. triangulosa (Walckenaer, 1802). Kopetdagh (Southwest and Central), Badghyz, Repetek. Holarctic species.
Thericlion pictum (Walckenaer, 1802). Kopetdagh (Southwest). EuropeanSiberian species.

T. simile C.L. Koch, 1836. Repetek. Ancient Mediterranean (trans-Palearctic?) species.

T. sisyphium (Clerck, 1757). Kopetdagh (Southwest). Trans-Palearctic species. T. varians (Hahn, 1831). Ashkhabad, Repetek. Trans-Palearctic species.

Fam. Lin!phiidae (= Erigonidae)

Agrnetu fuscipalpis (C.L. Koch, 1836). Kugitang (1,200-1,300 m), Repetek. European-Siberian species.

A. Kopetdcighensis Tanasevitch, 1989. Kopetdagh (Southwest: Aidere). Iranian species.

A. ressli (Wunderlich, 1973). Kopetdagh (Southwest). Mediterranean species. A. rurestris (C.L. Koch, 1836). Ashkhabad. European-Siberian species.

Aliorcums.s cuvanturus Andreeva et Tystshenko, 1970. Kugitang (1,400 m). IranoTurkestanian species.

Ceratinclla brevis (Wider, 1834). Kopetdagh (Southwest). European-Siberian species.

Diplocephalus hifurcatus Tanasevitch, 1989. Kopetdagh (Southwest). Iranian species.

Donacochara speciosa (Thorell, 1875). Badkhyz. European species.

Erigonc dentipalpis (Wider, 1834). Kugitang (1200-1300 m). EuropeanMediterranean-Siberian species.

Erigonoplus nince Tanasevitch et Fet, 1986. Kopetdagh (Southwest). Iranian species.

Frontinellina frutetorum (C.L. Koch, 1834). Kopetdagh (Southwest). EuropeanSiberian species (eastward to East Kazakhstan Region).

Gomglidicllum murciclum Simon, 1884. Kopetdagh (Southwest). European

species.
Jametschekia necessaria Tanasevitch, 1985. East Karakum (Farab). Turanian desert species.

Leptliyphante's badkhyzensis Tanasevitch, 1986. Badkhyz. Turanian desert

species.
L. escupus. Tanasevitch, 1989. Kugitang (1,200 m). Turkestanian species.

L. Kuhitangensis Tanasevitch, 1989. Kugitang (1,200-1,400 m). Turkestanian species.

L. nehulosoide's Wunderlich, 1977. Kopetdagh (Southwest), Kugitang. Ancient Mediterranean species.

L. pinicola Simon, 1884. Kopetdagh (Southwest). European-Mediterranean

Species.
L. I'muis Blackwall, 1852. Kopetdagh (Southwest and Central). EuropeanMediterranean species (eastward to Tajikistan).

l. turanicus Tanasevitch et Fet, 1986. Tuarkyr Plateau (Kafigshem Mts.), Kopetdagh (Southwest and Central), Kugitang (1,300 m). Iranian species. 
L. Iurkestanicus Tanasevitch, 1989. East Karakum (Farab). Turanian desert species.

Mecopisthes orientalis Tanasevitch et Fet, 1986. Kopetdagh (Southwest). Iranian species.

Mesasigone mira Tanasevitch, 1989. Kugitang $(1300 \mathrm{~m})$. Iranian species.

Microctenonly subitaneus (O. Pickard-Cambridge. 1875). Kopetdagh (Southwest and Central), Farab. Holarctic species (with a range disjunction in Siberia).

Microlinyphia pusilla (Sundevall 1830). Kopetdagh (Southwest). Ashkhabadd. Badghyz, Farab. Holarctic species.

Oedothorax apicatus (Blackwall, 1850). Kopetdagh (Southwest), Kugitang European species.

Pelecopsis laptevi Tanasevitch et Fet, 1986. Badghyz. Turanian desert species.

$P$. paralleloides Tanasevitch et Fet, 1986. Kopetdagh (Southwest). Iranian species.

Prinerigone v'agans (Audoin, 1826). Kopetdagh (Southwest and Central), Bakharden District, Ashkhabad District, Farab. European-Mediterranean (steppe?) species.

Sphecosone romana (O. Pickard-Cambridge. 1872).

C'ratinopsis romana O. Pickard-Cambridge, 1872.

Sphecozone asiatica (Andreeva et Tyschchenko, 1970) (Tanasevitch 1983: 1786).

Repetek. Mediterranean species.

Trachelocamptus asiaticus Tanasevitch, 1989. Repetek. Turanian desert species. Trichoncoides piscator Simon, 1884. Kopetdagh (Southwest). Mediterranean species.

Malckenaeria monoceros (Wider, 1834). Kopetdagh (Southwest). European species.

Firm. Tetragnathidae

Me'tellina kirgisica (Bachvalov, 1974).

Meta kirgisica Bachvalov 1974) (Marusik 1989).

Kugitang. Irano-Turkestanian species.

Tetragnatha extensa (L., 1758). Kopetdagh (Southwest). "Tekke". Holarctic species.

Zigiella caspica (Simon, 1889). Imambaba. Turanian desert species.

Farm. Araneidae

Aculepeira sogdiana (Charitonov, 1969). Kopetdagh (Southwest and Central), Badghyz. Turanian desert (or Iranian?) species.

Agalenatea redii (Scopoli, 1763). Kopetdagh (Southwest). EuropeanMediterranean species (from North Africa to Middle Asia).

Araneus angulatus Clerck, 1757. Kopetdagh (Southwest). European-
Mediterranean forest species. Records from the New World are erroneous!

A. armida (Auduin, 1825). Ashkhabad. European-Mediterranean species (present in the Caucasus).

A. bituberculatus (Walckenaer, 1802). Kopetdagh (Southwest and Central), Badghyz. European-Ancient Mediterranean species.

A. pallasi (Thorell, 1875). Kyzylsu (Krasnovodsk Region), next to the water. Turkestanian-Turanian desert species (eastward to Mongolia and Tuva).

A. repetecus Bachvalov, 1978. Repetek. Turanian desert species.

A. spasskı

A. crucificroicles Spassky 1952.

Kopetdagh (Southwest). Irano-Turkestanian species.

A. tartaricus (Kroneberg, 1875). Ashkhabad, Badghyz. Irano-Turkestanian species.

d. I'lycinicus Bachvalov, 1978. Tedzhen? (place of collection is absent from the original description). Turanian desert species.

Araniclla inconspicua (Simon, 1874). Kopetdagh (Southwest). EuropeanCaucasian (European-East Mediterranean?) meadow species.

Argiope almgeri Spassky, 1932. Akhal-Teke. Irano-Turkestanian species.

A. hruennichi (Scopoli, 1772). Kopetdagh (Southwest). Trans-Palearctic meadow-steppe species.

A. Lohata (Pallas, 1772). Krasnovodsk, Kopetdagh (Southwest and Central), Bakharden, Ashkhabad, Badghyz, Murghab, Takhta-Bazar. Paleotropical and Palearctic species (in Palearctic, occupies steppe habitats).

Ciclosa conica (Pallas, 1772). Kopetdagh (Southwest). Holarctic forest species. Hypsosinga albovittata (Westring, 1851). Kopetdagh (Southwest). TransPalearctic species.

H. Iurkmenica Bachvalov, 1978. Repetek. Turanian desert species.

Larinia nenilini Marusik, 1986. Repetek, Chardzhou. Turanian desert species. L. pubiventris Simon, 1889.

L. Iurkmenica Spassky 1939.

Krasnovodsk, Tedzhen, Kalaimor (= Mor-Kala), Imambaba, Repetek. Turanian desert species.

L.arinioidles folium (Schränk, 1803). Gasan-Kuli, Ashkhabad, "Tekke," Amudarya. European-Mediterranean steppe-desert species, found next to the water (eastward to Middle Asia).

Mangora acalypha (Walckenaer, 1802). Kopetdagh (Southwest). EuropeanAncient Mediterranean species; eastward to southern Urals, Tajikistan, Kyrghyzstan, and East Kazakhstan Region.

Neoscona adianta (Walckenaer, 1802). Kopetdagh (Southwest), Karakum District (Karakumkanal State Farm). Trans-Palearctic meadow-steppe species.

N. subfusca (C.L. Koch, 1837).

Arameus dalmaticus Doleschall 1852.

Kopetdagh (Southwest). Mediterranean species (the easternmost record!). 
Zilla diodia (Walckenaer, 1802). Kopetdagh (Southwest). European-Caucasian (meadow?) species.

\section{Fam. Ly'cosidae}

Arctosa soror (Simon, 1889). Mary ("New Merv"). Turanian desert species. A. variana (C.L. Koch, 1848). Badghyz. European-Mediterranean species. "Arctosa" cercipes (L. Koch, 1878). Krasnovodsk. Turanian desert species. Aulonia kratochili Dunin. Buchar, et Absolon. 1986. Kopetdagh. CaucasianIranian species.

Evippa badchysica Sternbergs, 1979. Badghyz. Turanian desert species.

E. onager Simon, 1895. Repetek. Sindian-Turanian desert (steppe) species (?).

E. schenkeli Sternbergs, 1979. Badghyz. Turanian desert species.

E. turkmenica Sternbergs, 1979. Badghyz. Turanian desert species.

Hippasa partita (O.Pickard-Cambridge, 1876).

H. deserticola Simon, 1889.

Murghab, Imambaba. Saharo-Sindian species.

L.jcosa alticeps Kroneberg, 1875. Krasnovodsk. Ashkhabad. Badghyz. Turanian desert species.

L. nordmanni (Thorell, 1875). Ashkhabad. Mediterranean species.

L. radiata (Latreille, 1819). Kopetdagh (Southwest and Central). Mediterranean species.

Pardosa italica Tongiorgi, 1966. Kopetdagh (Southwest and Central). Mediterranean species.

P. morosa (L. Koch, 1870). Kopetdagh (Southwest and Central). Mediterranean species.

P. nehulosi (Thorell, 1892). Kopetdagh (Southwest). Badghyz. Sultanbent. Iolatan. Mary (= New Merv). Mediterranean species.

P. pontica (Thorell, 1875). Kopetdagh (Southwest and Central). CaucasianIranian species (?).

P. proxima C. L. Koch, 1847. Kopetdagh (Southwest). EuropeanMediterranean species.

Tarentula albofasciata (Brullé, 1832). Kopetdagh (Southwest). Ashkhabad. Mediterranean species.

T. hergsoei (Thorell, 1875). Ashkhabad. Caucasian-Iranian species (?).

T. cromebergi (Thorell, 1875). Murghab District. Ancient Mediterranean species.

T. cursor (Hahn, 1831) cursorioides Charitonov. 1969). Kopetdagh (Southwest and Central). Iranian subpecies of an European-Ancient Mediterranean species.

T. ruddei (Simon, 1889). Mary ("New Merv"), Amudarya. Turanian desert species.

Trochosa ruricola (De Geer, 1778). Kopetdagh (Southwest). Trans-Palearctic species.

T. terricola Thorell, 1856. Kopetdagh (Southwest). Trans-Palearctic species.
Wadicosa commoventa Zyuzin, 1985. Badghyz (Kushka). Turanian desert species.

Xerolycosa brunneopicta Loksa, 1965. "Duldsch (Sudüfer d. Balchanbühels)", Krasnovodsk. Mongolian desert (steppe) species.

Fann. Piscurriclae

Pisaura mirabilis (Clerck, 1757). Kopetdagh (Southwest). EuropeanMediterranean species.

P. novicic (L. Koch, 1878). Ashkhabad. Caucasian-Iranian species (?). Taxonomic status in relation to $P$. mirabilis is not clear.

Farm. Ageleniclae

Agelena labyrinthica (Clerck, 1757). Kopetdagh (Southwest and Central). Trans-Palearctic species (eastward to Manchuria and Japan; southward to the Himalayas).

Tegenaria domestica (Clerck, 1757). Kopetdagh (Southwest and Central). Cosmopolitan species.

Fim. Desidue

Cedicus ephthalitus Fet, 1993. Known only from Southwest Kopetdagh. Iranian species.

C. gennadii Fet, 1993. Kopetdagh (Southwest and Central), Tuarkyr (Kafigshem Mountains). Iranian species.

c. muerens Simon, 1889. Known only from the foothills of Southwest Kopetdagh and Bolshoi Balkhan. Iranian species.

C. parthus Fet, 1993. Known only from Kopetdagh (Southwest and Central). Iranian species.

Fam. Halmiidace

Halmia sp. Kopetdagh (Southwest and Central). Iranian species.

\section{Finm. Dicrninidue}

Archacodictlyna ammophila (Menge, 1871). Kopetdagh. European-Caucasian meadow species.

A. consecuru (O. Pickard-Cambridge, 1872). Kopetdagh (Central). East Mediterranean speccies (from Middle East to Turkestan).

Brigitteu latens (Fabricius, 1775). Kopetdagh (Southwest). EuropeanCaucasian meadow species, eastward to Tajikistan and Kyrghyzstan.

Devade tenella (Tystshenko, 1965). Badghyz. Turanian desert (or steppe) species. 
Dictyna cronebergi Simon, 1889. Mary (= New Merv). Turanian desert species.

D. pusilla Thorell, 1856. Kopetdagh (Southwest). Trans-Palearctic species (eastward to Kamchatka).

D. uncinata Thorell, 1856. Ashkhabad. Trans-Palearctic species (eastward to Kamchatka).

Dictlyomorpha strandi Spassky, 1939. Meshed-Messerian Plain. Ashkhabad. Turanian desert species.

Fum. Amaurohiidue

Coclotes charitonovi Spassky, 1939. Krasnovodsk, Badghyz. Turanian desert species.

Finm. Titanoecidae

Titanoseca albomaculata (Lucas, 1846). Kopetdagh (Southwest). Mediterranean species.

T. Lehtinemi Fet. 1986. Kopetdagh (Southwest, Central, and East). Badghyz. Iranian species.

T. tristis (L. Koch, 1872). Kopetdagh. European-Caucasian meadow species (the easternmost record!).

T. veteranica (Herman, 1879). Kopetdagh (Southwest). Mediterranean steppe species.

Fam. Oxyopidae

Oryopes bully:icus Mikhailov et Fet, 1986. Known only from Badghyz (Lake Yeroyulanduz). Turanian desert species.

O. heterophthalmus Latreille, 1804. Ashkhabad. Bagir. European-Ancient Mediterranean species.

O. Lineutus Latreille, 1806. Kopetdagh (Southwest). Badghyz. EuropeanMediterranean species.

O. maracumlensis Charitonov, 1946. Kopetdagh (Southwest and (entral). Badghyz, Sultanbent, Murghab District, Karakum District. Repetek. Turanian desert species.

O. takohius Andreeva et Tystshenko, 1969. Kopetdagh (Southwest and Central), Badghyz. Iranian-Turkestanian species.

Fam. Anyphaenidare

Anyphuena accentuata (Walckenaer, 1802). Kopetdagh (Southwest). EuropeanMediterranean species.
Fam. Liocranidae

Agroeca pullata Thorell, 1875. Kopetdagh (Southwest and Central). EuropeanMediterranean-Siberian species.

Mesiotelus kulc:zynskii Charitonov, 1946. Kopetdagh (Southwest and Central). Iranian species.

M. temuissimus (L. Koch, 1866). Kopetdagh (Southwest and Central). Mediterranean species.

Orthohula charitonovi (Mikhailov, 1986) comb. novi by K. Mikhailov.

Trachelas charitonovi Mikhailov, 1986.

Kopetdagh (Southwest and Central). Eastern Mediterranean species (from the Caucasus to Kyrghyzstan).

Phrirrolithus pullatus Kulczynski, 1897. Kopetdagh (Southwest). Mediterranean species.

Fam. Clubioniclae

Cheirac'unthium ('raticum (Walckenaer, 1802). Kopetdagh (Southwest). TransPalearctic species.

C. mildei L. Koch, 1866. Kopetdagh (Southwest). European-Mediterranean species (introduced to the USA).

C. seicllitzi L. Koch, 1864. Kopetdagh (Southwest), Badghyz, Kalaimor (MorKala). European-Mediterranean species.

Clubiona alpicola Kulczynski, 1882. Kopetdagh (Southwest). European meadow species (the easternmost record!).

C. gene'e'nsis L. Koch, 1866. Kopetdagh (Southwest). Ancient Mediterranean species.

Fam. Zodariiclae

Zodurion prosz:1\%skii Nenilin et Fet, 1985. Kopetdagh (Southwest and Central). Iranian species.

7. rudldei Simon, 1889.

7. vasovi Sytshevskaya in Vlasov et Sytshevskaya, 1937.

Z. denisi: Ovtsharenko and Fet 1980, Krivokhatsky and Fet 1981; misidentification, non Z. denisi Spassky, 1938.

Z. raldei: Fet $1985 \mathrm{~b}$.

Kopetdagh (Southwest and Central), Archman, Ashkhabad, Badghyz, Repetek. Iranian species.

7. syrcherskayae Nenilin et Fet, 1985. Kopetdagh (East), Babadurmaz, Badghyz, Repetek. Turanian desert species. 


\section{Fam. Gnaphosidac}

Aphantaulax seminigra Simon, 1878. Kopetdagh (Southwest and Central). Mediterranean species (eastward to Kyrghyzstan).

Asiubadus asiaticus (Charitonov, 1946). Badghyz. Turanian desert species. Berlandina afghana Denis, 1958. Repetek. Turanian desert species.

B. caspica Ponomarev, 1979. Murghab District. Turanian desert species.

B. plumalis (O. Pickard-Cambridge, 1872). Badghyz. Mediterranean and Paleotropical (Burma. Himalayas, China) species.

B. sp. Kopetdagh (Central).

Drassodes jakkabagensis Charitonov, 1946. Badghyz. Turanian desert species

D. lupidosus (Walckenaer, 1802). Kopetdagh (Southwest and Central). TransPalearctic species.

D. proximus (Denis, 1958). Krasnovodsk, Kopetdagh (Central). I ranian species. D. sp. Kopetdagh (Southwest and Central). Iranian species.

"D." flavomaculatus (L. Koch, 1878). Krasnovodsk. Turanian desert species. Does not belong to the genus Drassodes (V. Ovtsharenko, pers. comm.).

"D. " thimei (L. Koch, 1878). Krasnovodsk. Turanian desert species. Does not belong to the genus Drassodes (V. Ovtsharenko, pers. comm.).

Echemus angustifrons (Westring, 1861). Kopetdagh. European species.

Ginaphosa hacarlovi Denis, 1958.

(i. ajidahamia Roewer, 1961 (Ovischarenko. Platnick, et Song. 1992.)

Ashkhabad Region (Gyuaurs). Irano-Turkestanian species.

(i. Kuld=ha Ovtscharenko, Platnick, et Song, 1992. Murghab District. IranoTurkestanian species.

(i. leporina (L. Koch, 1866). Kopetdagh (Southwest and Central). Ancient Mediterranean species.

G. Iurkmenica Ovtscharenko, Platnick. et Song 1942. Known only from Badghyz (Lake Eroyulanduz).

(i. lugubris (C.L. Koch, 1839). Record for Repetek (Kilplin 197א) is not confirmed (Ovtsharenko 't al. 1992).

Haplodrassus dalmatensis (L. Koch, 1866). Kopetdigh (Southwest and Central) European-Mediterranean species.

11. signifer (C.L. Koch, 1839). Kopetdagh (Southwest and Central). Aslikhabad, Badghyz. Holarctic species.

Micaria alhimama O. Pickard-Cambridge. 1872. Kopetdagh (Central and East). Badghyz, Repetek. East Mediterranean species.

11. koperdughensis Michailov in Mikhailov et Fet. 1986. Kopetdagh (Southwest). Caucasian-Iranian species.

M. lensi Bösenberg, 1899. Sarykamysh. Trans-Palearctic species.

M. p.'gmaea Kroneberg. 1875. Sharlouk. Ancient Mediterranean species (from Canary Islands to Tajikistan).

M. romana L. Koch, 1866. Kopetdagh (Southwest). European-Mediterranean species.

M. rossica Thorell, 1875. Kopetdagh (Southwest, Central, and East), Dushak,
Badghyz, Repetek, Farab, Tashauz Region (Chirishli, Kankakyr). Holarctic species.

M. septempunctata O. Pickard-Cambridge, 1872. Gasan-Kuli, Kopetdagh (Central), Bairam-Ali, Repetek, Farab. East Mediterranean species.

Minosia karakumensis (Spassky, 1939). Kopetdagh (Southwest and Central), Ashkhabad, Badghyz, Repetek. Turanian desert species.

Minosiella intermedia Denis, 1958. Kopetdagh (Southwest), Serakhs District, Badghyz, Repetek. Iranian species (?).

Nomisia aussereri (L. Koch, 1872). Kopetdagh (Southwest and Central), Ashkhabald. Mediterranean species.

N. conigera (Spassky, 1941). Kopetdagh (Southwest, Central, and East), Badghyz. I rano-Turkestanian species.

N. exornata (C.L. Koch, 1839). Kopetdagh (Southwest, Central, and East), Badghyz. Ancient Mediterranean species.

Poecilochroa conspicua (L. Koch, 1866). Ashkhabad,Repetek. EuropeanAncient Mediterranean species.

Prodiclomus redikorzevi Spassky, 1940. Kopetdagh (Southwest), Serakhs, Badghyz. Iranian species.

Pterotricha strandi Spassky, 1936. Kopetdagh (Southwest and Central), AkhalTeke, Serakhs District, Badghyz. Iranian species.

Scotophueus scutulatus (L. Koch, 1866). Kopetdagh. European species.

S! maphosus see Addendum (p. 524).

$S$. sp. 2. Badghyz. Iranian species.

Tulumites dlunini Platnick et Ovtsharenko, 1991. Kopetdagh (Southwest and East). Iranian species.

T. fugei Spassky, 1938. Kopetdagh (Central), Serakhs. Iranian species.

"Tulunites" aculeatus Charitonov, 1946. Kopetdagh (Southwest and Central), Repetek. Turanian desert species. Does not belong to the genus Talanites (V. Ovisharenko, pers. comm.).

"T." sp. Repetek. Turanian desert species. Belongs to the same genus as "Talcmite's" aculecatus Charitonov.

The'umu walteri (Simon, 1889). Kalaimor (= Mor-Kala). Turanian desert species.

Trachyzclotes jaxartensis (Kroneberg, 1875). Kopetdagh (Southwest), Badghyz. Ancient Mediterranean species (introduced to the USA, Mexico, India, China, Senegal, South Africa, and Hawaii).

Zelotes acrosus Charitonov, 1946. Kopetdagh (Southwest and Central). Turanian desert species.

7. arnoldii Charitonov, 1946. Ashkhabad, Meshed-Messerian Plain. Turanian desert species.

Z. bucharensis Charitonov, 1946. Kopetdagh (Central), Badghyz, Repetek. Turanian desert species.

Z. cauccusius (L. Koch, 1866). Kopetdagh (Central). Mediterranean species.

Z. Iongipes (L. Koch, 1866). Kopetdagh (Southwest). European-Siberian species. 
7. praeficus (L. Koch, 1866). Kopetdagh (Southwest and Central). EuropeanWest Siberian species.

7. pumilus (C.L. Koch, 1839). Kopetdagh (Southwest). EuropeanMediterranean species.

7. pusillus (C.L. Koch, 1833). Kopetdagh (Southwest and Central). TransPalearctic species.

Z. suhterraneus (C.L. Koch, 1833). Kopetdagh (Central), Repetek. Holarctic species.

Fam. Zoridae

Zora nemoralis (Blackwall, 1861). Kopetdagh (Southwest and Central). European species.

Z. silvestris Kulczynski in Chyzer et Kulczynski, 1897. Kopetdagh (Southwest). European species.

Fam. Heteropodidae $(=$ Sparassidae, $=$ Eus.sparassidue $)$

Cihr('nmus sp. Repetek. Turanian species.

linsparassus oculatus (Kroneberg, 1875). Kopetdagh (Southwest and Central), Archman, Ashkhabad, Badghyz. Repetek. Irano-Turkestanian species.

Micrommata ligurinum C.L. Koch, 1845. Kopetdagh (Southwest and Central). Mediterranean species.

Olios sericeus (Kroneberg, 1875). Kopetdagh (Southwest and Central), Ashkhabad, Badghyz, Kushka, Repetek. Irano-Turkestanian species.

Fum. Philodromidue

Paratihellus oblongiusculus (Lucas, 1846). Kopetdagh (Southwest), Bagir. Mediterranean species.

Philodrommes cureolus (Clerck, 1757). Kopetdagh (Southwest). Trans-Pallearctic species [?" - many records probably belong to $P$. cespitum (Walckenaler 1S(1)2)].

P. folltax Sundevall, 1832. Kopetdagh (Southwest). European species.

P. Iepidlus Blackwall, 1870. Mary (= New Merv). Uch-Adzhi. Mediterranean species.

P. rufus Walckenaer, 1825. Kopetdagh (Southwest). Holarctic species.

Thumatus formicinus (Clerck, 1757). Kopetdagh (Southwest and (entral). Holarctic species.

T. imbecillus L. Koch, 1878. Kopetdagh (Southwest), Archman. CaucasianTurkestanian species.

T. vulgaris Simon, 1870. Kopetdagh (Southwest and Central). Holarctic species. Tihellus oblongus (Walckenaer, 1802). Kopetdagh (Southwest). Ashkhabad. Holarctic species.
Fulm. Thomisidale

Diala dorsala (Fabricius 1777). Kopetdagh (Central) (the record is dubious). European-Ancient Mediterranean species.

Heriucus buffonopsis Loerbroks, 1983. Krasnovodsk. Turanian desert species.

II. fedotovi Charitonov, 1946. Kopetdagh (Southwest). Turanian desert species. H. mellottei Simon, 1886.

II. oblongus Simon, 1918.

Kopetdagh (Southwest). European-Ancient Mediterranean species.

11. spinipalpus Loerbroks, 1983. Krasnovodsk, Firyuza. CaucasianTurkestanian species.

Monacse's israilensis Levy, 1973. Kopetdagh (Southwest). East Mediterranean

M. paradoxus (Lucas, 1846). Kopetdagh (Southwest), Geok-Tepe, Repetek. Mediterranean-Afrotropical species.

Oxiptila handucri cribrata (Simon, 1885).

Xi:sticus cribratus Simon, 1885.

P.summititis cribratus (Simon, 1885).

Kopetdagh (Southwest and Central). Synonymy of this Mediterranean form is not clear. Kritscher (1961) placed it as a subspecies into $O$. baudueri Simon, 1877.

O. sanculuria (O. Pickard-Cambridge, 1871). Kopetdagh (Southwest). European species.

O. Iricoloripes Strand, 1913. Kopetdagh (Southwest and Central). East Mediterranean species.

"O". lugibris (Kroneberg, 1875). Kopetdagh (Southwest and Central), Ashkhabad, Repetek. Irano-Turkestanian species; belongs neither to the genus Oxyptila nor to Xysticus (Marusik and Logunov, 1990: 52).

Penumitis tristrami (O. Pickard-Cambridge, 1872). Durun, Bairam-Ali. Mediterranean species.

P. Aurunicus (Charitonov, 1969), comb. novi.

Yisticus murcunicus Charitonov, 1969.

Kizyl-Atrek, Sharlouk, Kopetdagh (Southwest and Central), Bakharden District, Badghyz. Irano-Turkestanian species.

Runcinic latcralis (C.L. Koch, 1838). Kopetdagh (Southwest). EuropeanMediterranean species.

Stiphropus strundi Spassky, 1938. Kopetdagh (Southwest and Central), Badghyz, Repetek. Irano-Turkestanian species.

Sinema globosum (Fabricius, 1775). Kopetdagh (Southwest). Trans-Palearctic species.

S. plorator (O. Pickard-Cambridge, 1872). Kopetdagh (Southwest). Mediterranean species.

Thomisus omustus. Walckenaer, 1805. Krasnovodsk, Kopetdagh (Southwest and Central), Ashkhabad, Gaudan, Bagir, Badghyz, Islimcheshme, Imambaba. Mediterranean-Paleotropical species. 
Xysticus acerhus Thorell, 1872. Kopetdagh (Southwest and Central). Ashkhabad, Repetek. Trans-Palearctic species.

X. caperatus Simon, 1875. Badghyz. Mediterranean species.

Y. caspicus Utotchkin, 1968. Kopetdagh (Southwest), Repelek. Turanian desert species.

X. concinnus Kroneberg, 1875. Badghyz, Kopetdagh (Central and East). Turanian desert species.

r. Kaznakovi Utotchkin. 1968. Kopetdagh (Southwest). Irano-Turkestanian species.

X. Kochi Thorell. 1872. Kopetdagh (Southwest). European-Mediterrancin species.

X. lapidarius Utotchkin. 1968. Iolatan. Irano-Turkestanian species.

X. marmoratus Thorell, 1875. Kopetdagh (Central). Mediterranean species.

X. minor Charitonov, 1946. Kopetdagh (Southwest and Central), Ashkhabad, Badghyz, Repetek. Irano-Turkestanian species.

X. nimnii Thorell, 1872. Kopetdagh (Southwest). European-Mediterrancan species.

\section{Famm. Salticidae}

Aelurillus andre'eve Nenilin, 1984: nomen novium for specimens misidentified as A. variegatus (Kroneberg, 1875) by Andreeva (1976). Ashkhabad. IranoTurkestanian species.

A. affinis (Lucas, 1846). Ashkhabad. Ancient Mediterrancan species.

A. ale'r (Kroneberg, 1875). Ashkhabad, Imambaba, Kaplankyr. Turanian desert species.

A. concolor Kulczynski, 1901.

A. iramus Roewer, 1955 (Nenilin 1984).

Kopetdagh (Southwest and Central), Murghab District. CattcasianTurkestanian (East Mediterranean?) species.

A. M-nigrum (Kulczynski in Chyzer et Kulczynski, 1891). Galsan-Kuli, Kopetdagh (Southwest and Central), Badghyz. Ancient Mediterranean species (eastward to Sinkiang).

A. variegatus (Kroneberg, 1875). Badghyz. Turanian desert species.

A. P-insignitus (Clerck, 1757). Kopetdagh (Southwest). European-Siberian species.

Ballus chalpheins (Walckenater, 1802). Kopetdagh (Southwest). I:uropeanMediterranean species (eastward to Tajikistan).

Biamor albomaculatus (Lucas, 1846). Bakharden. Ancient Mediterranean species.

Chalcoscirtus infimus (Simon, 1868). Kopetdagh (Southwest and Central). European-Mediterranean species.

C. martensi parvus Marusik, 1991. Kopetdagh (Southwest). Irano-Turkestanian subspecies.
Cyrha algerina (Lucas, 1846). Kopetdagh (Southwest). Mediterranean and Paleotropical species.

C. ocellata (Kroneberg, 1875). Kopetdagh (Southwest and Central), Repetek. Mediterranean and Paleotropical species.

Eris nidicolens (Walckenaer, 1802). Kopetdagh (Southwest). EuropeanMediterranean species.

Euophrys frontalis (Walckenaer, 1802). Kopetdagh (Southwest). TransPalearctic species.

Evarcha arcuata (Clerck, 1757). Kopetdagh (Southwest), Repetek. TransPalearctic species.

Ileliophamus aturatus C. L. Koch, 1835. Lower Murghab. European-Siberian species.

H. curvidens (O. Pickard-Cambridge, 1872). Bakharden. East Mediterranean species.

II. flavipes (Hahn, 1832). Kopetdagh (Southwest). European-Siberian species.

II. lineiventris Simon, 1868. Badghyz. Trans-Palearctic species.

II. melimus L. Koch, 1867. Kopetdagh (Southwest). Ancient Mediterranean species.

H. niveivestis Simon, 1889. Bairam-Ali (= Old Merv). Turanian desert species. Taxonomic status is not clear.

11. patagiatus Thorell, 1875. Exact locality in Turkmenistan is not published (A. Nenilin, personal notes). European-Siberian species.

H. Iurcunicus Charitonov, 1969. Kopetdagh (Southwest), Khozli-ogly-olum (collected by K. Ahnger, November 1 to 11, 1903). Turanian desert species. Langona tartarica (Charitonov, 1946). Kopetdagh (Central). IranoTurkestanian species.

Leptorchestes berolinensis (C.L. Koch, 1846). Kopetdagh (Southwest). European-Mediterranean species.

Menemerops afghanus (Roewer, 1961). Kopetdagh (Southwest), Badghyz. Iranian species.

Menemerus marginatus (Kroneberg, 1875). Sultanbent, Repetek. IranoTurkestanian species (northwestward to Nakhichevan).

Mogrus antonimus Andreeva, 1976. Kopetdagh (Southwest and Central), Ashkhabad, Badghyz. Irano-Turkestanian species.

M. valerii Kononenko in Andreeva, Kononenko, et Proczynski, 1981. Repetek. Turanian desert species.

Pellene's c'pularis (O. Pickard-Cambridge, 1872). Exact locality in Turkmenistan is not published (A. Nenilin, personal notes). East Mediterranean species.

P. limbatus Kulczynski, 1895. Ashkhabad (Berzengi), Badghyz. TurkestanSiberian species.

P. kulabicus Andreeva, 1976. Ashkhabad, Badghyz. Irano-Turkestanian species.

P. nigrociliatus (L. Koch, 1875). Kopetdagh (Southwest). EuropeanMediterranean and Japanese (possibly Trans-Palearctic) species. 
P. simoni (O. Pickard-Cambridge, 1872). Exact locality in Turkmenistan is not published (A. Nenilin, personal notes). East Mediterranean species.

P. Iripunctatus (Walckenaer, 1802). Kopetdagh (Southwest). EuropeanSiberian species.

Philaeus chrysops (Poda, 1761). Kopetdagh (Southwest and Central), Ashkhabad, Serakhs, Badghyz. European-Ancient Mediterranean species (eastward to Sinkiang).

Phlegrafasciata (Hahn, 1826). Kopetdagh (Southwest). Ancient Mediterranean species (eastward to Sinkiang).

P. sogdiana Charitonov, 1946. Kopetdagh, Kugitang. Irano-Turkestanian species.

Plexippoides starmuehlneri (Roewer, 1955). Murghab Oasis. Iranian species.

Plevippus coccineus Simon, 1902. Kopetdagh (Southwest and Central). Badghyz. Ancient Mediterranean (Caucasian-Iranian?) species.

P. sertipes (Karsch, 1879). Krasnovodsk. Paleotropical species (from Turkestan (1) Vietnanı).

Pse'mulicius c'inctus (O. Pickard-Cambridge, 1872).

P. villatus Simon, 1889.

Krasnovodsk, Kopetdagh (Southwest). Badghyz. Murghab Oasis. SaryYazy, Repetek. Irano-Turkestanian species (eastward to Sinkiang).

P. spasskyi Andreeva, Heçiak et Proczynski, 1984. Krasnovodsk. Murghab District. Irano-Turkestanian species.

Salticus tricinctus (C.L. Koch, 1846). Krasnovodsk, Badghyz (Kushka). East Mediterranean species.

Sitticus caricis (Westring, 1861). Farab. European-Siberian (?) species. S. distinguendus (Simon, 1868). Krasnovodsk, Kopetdagh (Central). Repetek.
European species.

S. Karakumensis Logunov, 1992. Badghyz. Repetek. Turanian desert species.

S. I'rehratus (Clerck, 1757). Locality in Turkmenistan is not published (Proczynski 1991). European-Siberian (?) species.

S. zimmermami (Simon, 1877). Kopetdagh (Southwest). European species.

Sinugeles charitonovi Andreeva, 1976. Murghab Oasis. Irano-Turkestanian species.

S. hilurulus (C. L. Koch, 1846). Exact locality in Turkmenistan is not published (Azheganova 1968). European species (Proczynski 1991).

S. ramitus Andreeva, 1976. Badghyz. Irano-Turkestanian species (eastward to Mongolia).

Thy'ene imperialis (Rossi, 1846). Kopetdagh (Southwest). Sharlouk. Sultanbent, Gasan-Kuli. Mediterranean-Paleotropical species.

Ylle'mus albocinctus (Kroneberg, 1875). Deinau. Caucasian-Mongolian species. $Y$. anspex (O. Pickard-Cambridge, 1885). Repetek. Turan-Mongolian desert
species. $Y$. hajam Proczynski, 1968. Krasnovodsk, Repetek. Turan-Mongolian desert
and steppe species.

Y. hamifer. Simon 1895.
Y. flarociliatus Simon, 1895.

"Southern Turkmenistan," Repetek. Turan-Mongolian desert and steppe species.

Y. mongolicus Proczynski, 1968. Repetek. Turan-Mongolian desert and steppe species.

Y. Imivitlatus Simon, 1871. Sultanbent, Repetek. Ancient Mediterranean species.

Y. valiclus Simon, 1889. Badghyz, Bairam-Ali (= Old Merv), "Bewüste Murghab." Irano-Turkestanian species.

Y. vittatıs Thorell, 1875. Kaplankyr. Mediterranean species.

\section{Zoogeographic Connections of the Spider Fauna of Turkmenistan}

The spider species listed above belong to a wide assemblage of genera and families with various ecological requirements. Due to their adept dispersal abilities (including the well-known "ballooning" of young spiderlings on silk threads), spiders commonly are not believed to be a very useful group of animals for a zoogeographic analysis. Nevertheless, we can detect certain patterns in their distribution within Turkmenistan and thus, will attempt to reveal zoogeographic connections of the local araneofauna. Of course, these conclusions will be subject to change when more knowledge is gained about distribution of spider species.

More than half of the entire faunal list (184 species, or $54.9 \%$ ) comprises spiders whose range within the Palearctic Realm is limited to one or a few biogeographic provinces. These species can be classified into several faunistic complexes which show current species distribution (and may also reflect centers of origin).

Iranian and Caucasian-Iranian species. Within Turkmenistan, these species are found in Kopetdagh (including Badghyz). Iranian species (38 spp., or $11.3 \%$ of the fauna) are Phyxioschema raddei, Nemesia birulai, Raveniola fedotovi, R. kope'daghensis, R. redikorzevi, Filistata sp., Ceratopholcus maculipes, Pholcus nenjukovi, Dysdera transcaspica, Palpimanus sogdianus, Hersiliola afghunica, H. sp., Pelecopsis paralleloides, Cedicus ephthalitus, C. gennadii, $C$. parthus, C. macrens, Agyneta kopetdaghensis, Diplocephalus bifurcatus, Erigonoplus ninae, Lepthyphantes turanicus, Mecopisthes orientalis, Mesasigone mira, Hahnia sp., Titanoeca lehtineni, Zodarion proszynskii, Z. radkei, Drassodes proximus, D. sp., Berlandina sp., Minosiella intermedia, Prodidomus redikorzevi, Pterotricha strandi, Synaphosus sp., Talanites dunini, T. fagei, Menemerops afghanus, and Plexippoides starmuehlneri. The five Caucasian-Iranian species are Aulonia kratochvili, Tarentula bergsoei, Pardosa pontic'a, Pisaura novicia, and Micaria kopetdaghensis.

Iramo-Turkestanian Species. These spiders are more widely found than Iranian 
ones and inhabit mountains and foothills of Middle Asia (including (hinese Turkestan), Afghanistan, and Iran. This group includes 36 species (10.7\% of the

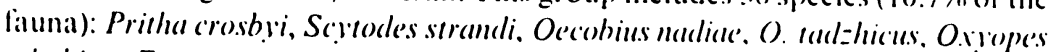
takobius, Eusparassus oculatus, Olios servicens, Alioramus avanturus. Mertellina kirgisica, Arameus spasskyi, A. tartaricus, Argiope ahmgeri. Gmaphosa haurlovi. (; kuld=ha. Nomisia conigera. Tarentula cursor cursorionides. "O)ryprila" lugihris,





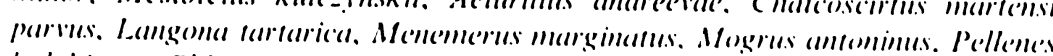


charitomovi, S. ramitus, and Yllemus valides. Some Turkestanian species, such as


kuhitangensis, and Mesasigone mira, are lound in Turkmenistan only in the Kugitang mountains (an offspur of the Pamiro-Alai range). Three species, Thanatus imbecillus, Heriaeus spinipalpus, and Aelurillus comcolor, are found from the Caucasus to Turkestan.

European and European-Cancasian Species. These are spiders of mesophilic habitats; in Turkmenistan. they are found only in the mountains of Kopetdagh. which often represent the easternmost limit of their distribution. The European group includes 16 species $(4.8 \%$ of the fauna): Enoplogmatha thoracica.

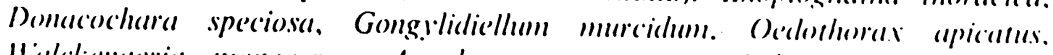

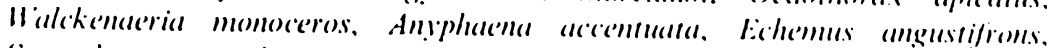
Scotophacess scutulatus, Clubiona alpicola. Zoma nemoralis. Z. silve'stris.

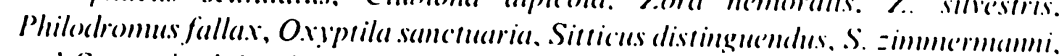
and Synageles hilarulus. Spiders of the European-Catucisian group include five species: Araniella inconcpicua, Zilla diodia, Archasedict!na ammonohila. Brigitlea latens, and Titanoeca tristis.

East Mediterrane'an Species. This small group includes 11 species: Latrodedens


Micaria septempunctata, Oryptila tricoloripes. Momacses israilensis.

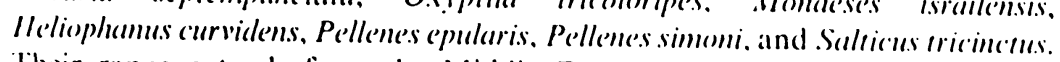
Their range extends from the Middle East 10 Kopetdagh but not further cilstward.

Tirranian Desere Spereics. These species inhabit the lowland deserts of Karakum and Kizylkum in Middle Asia, may penetrate into semidesert foothills but are not found in mountainous habitats. Some of these species are aldopted to sand desert habitats and are specialized psammophiles (e.g.. Sitticus kurakume'nsis): many inhabit rodent burrows (e.g., Minosiella intermedic, Krivokhattsky and Fet 1982). The Turanian desert group, which includes 58 spp.. or $17.3 \%$ of the total fauna, comprises the following: Artema transcaspica, Disclera aculeata, $D$. limitanea, D. pococki, Hersiliola sp., Janctschekia necessaria, Lepthyphantes balkhyzensis, L. turkestanicus, Pelecopsis laptevi. Trachelocamptus asiaticus,
Acule'peira sogdiana, Araneus repetecus, A. tedgenicus, Hypsosinga turkmenica, Larinia nenilini, L. pubiventris, Zygiella caspica, "Arctosa" cereipes, Tarentula radlei, Arctosa soror, Evippa badchysica, E. schenkeli, E. turkmenica, Lycosa alticeps, Wadicosa commoventa, Devade tenella, Dictyna cronebergi, Dict!nomorpha strandi, Coelotes charitonovi, Oxyopes badhyzicus, $O$. maracandensis, Zodarion sytchevskayae, Asiabadus asiaticus, Berlandina af.ghana, B. c'aspica, Drassodes jakkabagensis, "D. "flavomaculatus, "D. " thimei, Ginaphosa turkmenica, Minosia karakumensis, Synaphosus sp., "Talanites" aculeatus. "T.".sp., Theuma walteri, Zelotes aerosus, Z. arnoldii, Z. bucharensis, Heriae'us buffonopsis, H. fedotovi, Xysticus caspicus, X. concinnus, Aelurillus at('), Heliophamus niveivestis, H. turanicus, Mogrus valerii, and Sitticus karakume'nsis. Related to this group are Turanian-Mongolian spiders that are found also larther to the east, in Mongolian deserts (Xerolycosa brunneopicta, Yllenus (auspex, Y. bajan, Y. hamifer, and Y. mongolicus), as well as Hippasa partila, which ranges from the Sahara Desert to the Sind, and Evippa onager, which is found from the Sind to Turan.

Our approximation shows that faunistic complexes of spiders found in Turkmenistan are roughly divided into mountain semiarid and humid fauna (species with European, European-Mediterranean, Iranian, IranoTurkestanian and related types of ranges) and arid fauna of lowland deserts (species with Turanian and related types of ranges).

The remainder of the araneofauna (150 spp., or $44.8 \%$ ) includes spider species which are broadly distributed within the Palearctic Realm or even more broadly. Several Holarctic species, spiders with Paleotropic connections, and a few cosmopolitan species are found in Turkmenistan. The majority of broadly distributed species are, however, those of the southern Palearctic, or so-called Ancient Meditcranean area, with all kinds of ranges, often including Europe or its southern part. Most of these species in Turkmenistan are found only in the mountains but not in the Karakum Desert. These include, for example, Mediterranean or European-Mediterranean species Atypus muralis, Scytodes bertheloti, Dysclerina loricata, Enoplognatha testacea, Agyneta ressli, l.'pthyphantes pinicola, Sphecozone romana, Trichoncoides piscator, Agalenatea redii, drancens angulatus, A. armida, Larinioides folium, Mangora acalypha, Neoscona subfisca, Titanoeca albomaculata, T. veteranica, Pisaura mirabilis, Taremtula albofasciata, Arctosa variana, Lycosa nordmanni, L. radiata, Pardosa italica, P. morosa, P. nebulosa, Cheiracanthium mildei, C. seidlitzi, Mesiotelus t'nuissimus, Phrurolithus pullatus, Haplodrassus dalmatensis, Aphantaulax sc'minigra, Micaria romana, Nomisia aussereri, Zelotes caucasius, Z. pumilus, Paratibellus oblongiusculus, Philodromus lepidus, Runcinia lateralis, Synema plorator. X!sticus caperatus, X. kochi, X. marmoratus, X. ninnii, Oxyopes linc'atus, delurillus affinis, A. m-nigrum, Ballus chalybeius, Chalcoscirtus infimus, Eris nidicolens, Leptorchestes berolinensis, and Yllenus vittatus. All these spiders are mesophilic or xeromesopilic, but definitely not adapted to extreme desert conditions. Their dispersal must have been prevented by deserts in at least the last two or three million years. Thus, whatever the origin of a broadly 
distributed species, it must have migrated through the mountains of Middle A sia in order to disperse along the southern Palearctic region.

In Turkmenistan, mountains and desert are divided only by a narrow (10 to 2() $\mathrm{km}$ ) belt of the arid foothills. The biogeographic border between the Mediterranean-type mountains of Kopetdagh and the lowland continental desert of Karakum is one of the best expressed (and least studied) ecological and hiogeographic boundaries that exists on the earth (Kryzhanovsky 1965). Over its geological history, Kopetdagh could have served as a biogeographic corridor for spider dispersal. In the Late Oligocene-Miocene (25 to 10 million years ago). reduction of the ancient Tethys Sea revealed an island/peninsular chain from the modern Balkans to the Armeno-Iranian Plateau. This chain was at natural corridor for dispersal (Kryzhanovsky 1965), as many island chains are today (e.g., the Antilles or Sunda Islands). With aridization continuing from the Eocene through the Oligocene, landscapes gradually changed. By the MiocentePliocene (from 5 to 2 million years ago), the mountain uplifi and receding Tethys Sea aridization separated deserts of Middle and Central $A$ sia from those of Sind, the Middle East, and North Africa and promoted the vicariant speciation on desert lowlands. However, the mountain chains of Zagroz. F:Ihurz, and Kopetdagh still could have served as effective dispersal routes. The sublatitudinal position of this uplifting mountain chain suggests that migrations of animals through it eastward or westward was not limited by latitudinal climatic changes (a common problem in such well-known dispersal cases as, for example. North/South American exchange). Moreover, the altitudinal zonation in mountains allowed dispersal of ecologically different animals migrating within specilic mountain belts. Located between northern and southern deserts of Asia, these mountains could house mesophile fauna which could not survive aridization of adjacent lowlands.

In the Pleistocene (less than one million years ago), the lrantan mountain corridor undoubtedly was a site of constant dispersal and, probably, also of locial speciation. Many local endemic species probably are of this alge. especially plant species (Kamelin 1970. 1979). Transgressions of the Proto-Caspian Sea periodically returned these desert mountains to the island condition.

During the recent glaciation ( 16,000 to 10,000 years ago) this corridor could have been invaded by almost modern European and Asian "refugee" species. Then, a new aridization disrupted many ranges and eflectively isolated European forest and meadow species in mountain valleys such as the Aidere Vallev in Southwest Kopetdagh. Spider fauna in walnut and elm forests of this magnilicent (ca. $30 \mathrm{~km}$ long) gorge bears strong European features and includes

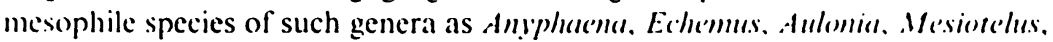

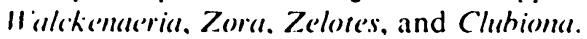

lixtensive local vicariant speciation (well demonstrated, for example, in numerous plant genera) along mountain systems from the (atucasus to Turkestan is observed also in spiders. For instance, a mygalomorph genus Ruveniolu Zonshtein, 1987 inhabits mountain forests at elevations above 1,000 $m$ and has several vicariant species from Georgia to the Himalayas, including $R$. koperdughensis from Southwest and Central Kopetdagh (Fet 1984). Another example is spider genus Zodarion, which has numerous endemic species throughout the Ancient Mediterranean area, with three species found only in Turkmenistan. Of these, Z. radde is a very common ant-eating spider in all arid habitats, including sand desert (where it is one of the common inhabitants of rodent burrows; Krivokhatsky and Fet 1982), foothills (Krivokhatsky and Fet 1981: Fet 1983), and mountains (Fet 1985b). Two other species, Zodarion pros=inskii and Z. sytchevskayae (Nenilin and Fet 1985), are vicariant in mountain valleys and desert foothills of Kopetdagh respectively. A third example of local vicariant speciation in Kopetdagh is a recently described (Fet 1993) subgenus Paracedicus belonging to the genus Cedicus. It includes two closely related species, Cedicus (Paracedicus) ephthalitus, which is found in the mountain valleys and humid meadows, and $C$. $(P$.) gennadii, found in semiarid mountain habitats.

Addressing the problem of local endemics (those of the Kopetdagh Mountains or Karakum Desert), we should remember that southern Palearctic spiders are still poorly studied. Within the Middle Asian republics of the former USSR, special araneological studies have been conducted only in Tajikistan and Turkmenistan; araneofauna of Uzbekistan, Kyrghyzstan, and Kazakhstan is poorly known, as are faunas of adjacent Afghanistan and, especially, Iran. In other areas of the Middle East and Central Asia, only faunas of Israel and India are known to some extent; no serious studies of spiders from Syria, Iraq, Pakistan, or Mongolia exist. Therefore, it is not practical to treat most spider species known presently only from Turkmenistan as endemics of local level. Mountain species may, in the future, be found within the Iranian or Turkestanian mountain systems, and desert spiders may turn out to be widespread Turanian desert elements. Nevertheless, examples from many other groups of animals and plants, some with good dispersal abilities, suggest that local endemism may be found among Turkmenistan spiders. As possible candidates for Kopetdagh endemics, we can list several species of the Iranian faunistic complex which are found so far only in the humid mountain forests of Kopetdagh: Raveniola kopetdaghensis, Cedicus ephthalitus, Hahnia sp., Zodurion proszynskii, Erigonoplus ninae, Mecopisthes orientaliis, and Drassodes sp. A few spider species, discovered so far only in the sand desert of Karakum (c.g., Sitticus kurakiumensis, Mogrus valerii, Cebrennus sp., and "Talanites" sp.), may belong to the Karakum psammophile endemics, which are quite common in most groups of animals and plants. The narrow belt of sagebrush-covered, loess foothills of Turkmenistan that separates desert from the mountains may also have been a site of speciation in spiders. Among species found only in these habitats (including the hilly plateau of Badghyz) are, e.g., Nemesia birulai, Rar'eniola redikorze'vi, Hersiliola sp., Pelecopsis paralleloides, Cedicus maerens, Titanoc'a lehtineni, Minosia karakumensis, Prodidomus redikorzevi, and Zodarion sitcherskciscue. Only from the salty depressions of Baghyz are known Oryopes badhyzicus and Gnaphosa turkmenica. The long and complicated geological history of landscapes of Turkmenistan has provided a diverse arena 
for differentiation of all ecological forms of spiders, from psammophiles of sand desert to mesophiles of mountain meadows.

\section{Acknowledgements}

We thank all the people who contributed to spider collection and helped in the lield trips in Turkmenistan, and, first of all, we give thanks 10 Gennady T. Kuznetsov, Svyatoslav I. Zabelin, Nina S. Ustinova, Lyudnila A. Mitroshina, Tamara M. Telyushenko, and Galina N. Fet. Rostislav A. Danov (1941-1993) and Yuri K. Gorelov were always helpful with their advice on natural history and biogeography as well as with their aid in field logistics. Identifications of many spider species were conducted or checked at different times by our colleagues Andrei B. Nenilin (1960-1986), Vladimir I. Ovtsharenko. Sergei L. Zonshtein, Alexei A. Zyuzin, Andrei V. Tanasevich, Yuri M. Marusik, Kirill Yu. Eskov, and Pyotr M. Dunin. Vladimir I. Ovtsharenko kindly helped to clarify recent synonymy of Gnaphosidae from Turkmenistan. We also thank for their constant support of our araneological studies Norman I. Platnick. Jerzy Proszynski, Gershom Levy, Pekka T. Lehtinen, Seppo Koponen. Alexander B. Lange, Yuri S. Balashov, Mikhail V. Heptner. Yaroslav I. Starobogatov, and Gary A. Polis.

\section{Addendum to the List of Spiders of Turkmenistan}

\section{Fam. Dysderidae}

Di:sdera brignolii Dunin, 1989. Repetek. Turanian desert species.

D. Kusnetsovi Dunin. 1989. Central Kopetdagh. Iranian species.

D. nenilini Dunin, 1989. Tuarkyr, Kaplankyr. Turanian desert species.

D. t!stshenkoi Dunin, 1989. Southwest Kopetdagh. Iranian species.

Harpactea parthica Brignoli, 1980. Krasnovodsk. Iranian species.

\section{lam. Gnaphosidae}

Sinaphosus karakimensis Ovtsharenko. levy et Platnick, 1994, Repetek.

Turanian desert species.

S. palearcticus Ovtsharenko, Levy et Platnick. 1994. Kopetdagh (Central). Ashkhabad, Badghyz, Mary, Chardzhou, Amudarya Valley. Ancient Mediterranean species.

S. soy'unovi Ovtsharenko, Levy et Platnick, 1994. Sarykamysh, Tashatuz Region. Turanian desert species.

S. turanicus Ovtsharenko, Levy et Platnick, 1994. Kopetdagh (Central). Tashauz Region. Ashkhabad, Badghyz, Mary, Repetek, Amudarya Valley. IranianTuranian species.

\section{Fauna and Zoogeography of Scorpions (Arachnida: Scorpions) in Turkmenistan}

\section{VICTOR FET}

\section{Abstract}

Scorpion fauna in Turkmenistan includes six genera and seven species of Buthidac, all belonging to the Saharo-Gobian (desert Palearctic) genera. Mc'sohuthus cupeus, M. caucasicus, and Orthochirus scrobiculosus are nearly ubicquitous and exhibit intraspecific variation; they are also widespread beyond the boundaries of Turkmenistan. Specialized Turanian sand desert genera include Liohuthus kessleri and Anomalobuthus rickmersi, and the endemic Pectinibuthus birulai. The last species, Kraepelinia palpator, is found in Turkmenistan only at salt lake shores (Yeroyulanduz Depression in the Badghyz Reserve); it is also known from Iran. Scorpion fauna of lowland Turkmenistan is a combination of widespread Asian and endemic Turanian and Iranian desert elements. There are no endemic species in the mountains; only one species, Mesobuthus eupeus, is commonly found in Kopetdagh.

\section{Introduction}

The fauna of scorpions of the former Russian Empire/USSR (i.e., primarily that of Caucasus and Middle Asia) was reviewed by Birula $(1911,1917 \mathrm{a}, \mathrm{b})$ and Fet (1990). In preparing this paper. we used data on scorpion fauna and ecology of Turkmenistan and adjacent countries published by the author (Fet 1980, 1984. 1987a.b. 1990) and other researchers (Simon 1889: Pocock 1899: Radde 1899: Morits 1922; Shestoperov 1934, 1935; Pavlovsky 1934; Vlasov 1937a,b; Vachon 1958a,b, 1966, 1974; Sabirova 1977, 1981, 1986). The largest museum collections reviewed by the author included those of the Zoological Institute of the Russian Academy of Sciences (St. Petersburg, Russia) and the Zoological Museum of Moscow State University, Moscow, Russia). Detailed data on museum collections, complete synonymy of Turkmen species and subspecies of scorpions, and complete references have been published (Fet 1990). 